\title{
Trends on physical understanding of bioink printability
}

\author{
Jun Yin ${ }^{1,2} \cdot$ Dengke Zhao ${ }^{1,2} \cdot$ Jingyi Liu ${ }^{1,2}$
}

Received: 20 January 2019 / Accepted: 23 January 2019 / Published online: 26 February 2019

(c) The Author(s) 2019

\begin{abstract}
Recently, 3D bioprinting is developed as an emerging approach, increasingly applied to materials for healthcare; while, the precise placement of cells and materials, and the shape fidelity of forming constructs is of great importance for successful application of 3D bioprinting. Research efforts have been made to develop new bioinks as "raw materials" with better biocompatibility and biofunctionality, but the printability of bioinks is largely ignored and still needs to be carefully examined to enable robotic bioprinting. This article aims to introduce a recent published review (Appl. Phys. Rev. 2018, 5, 041304) on the evaluation of bioink printability by Huang's research group from University of Florida. Huang et al. comprehensively reviewed the bioink printability based on the physical point of view during inkjet printing, laser printing, and microextrusion, and a series of self-consistent time scales and dimensionless quantities were utilized to physically understand and evaluate bioink printability. This article would be helpful to know the trends on physical understanding of bioink printability.
\end{abstract}

Keywords Bioink $\cdot$ 3D bioprinting $\cdot$ Printability $\cdot$ Timescale $\cdot$ Dimensionless numbers $\cdot$ Phase diagram

Over the decade, additive manufacturing (3D printing) has been established as the most promising technology for material processing. Recently, 3D bioprinting is developed as an emerging approach, increasingly applied to materials for healthcare [1]. 3D bioprinting aims to fabricate sophisticated constructs for a variety of applications including tissue engineering, regenerative medicine, drug testing and screening, physiological/pathological modeling [2]. As such, precise placement of cells and materials and the shape fidelity of forming constructs are of great importance for successful application of 3D bioprinting. The "raw materials" used in 3D bioprinting are referred as bioinks, which may include living cells, extracellular matrix materials, cell media, and other additives. So, the ability of printing bioinks rather than thermoplastic and metal inks leads to the booming of bioink research. However, recent bioink research efforts have been made to develop new bioink materials for better

Jun Yin

junyin@zju.edu.cn

1 The State Key Laboratory of Fluid Power and Mechatronic Systems, School of Mechanical Engineering, Zhejiang University, Hangzhou 310028, China

2 Key Laboratory of 3D Printing Process and Equipment of Zhejiang Province, School of Mechanical Engineering, Zhejiang University, Hangzhou 310028, China biocompatibility and biofunctionality; the printability of bioinks is largely ignored and still needs to be carefully examined to enable robotic bioprinting.

Huang's research group from University of Florida recently published a review on the evaluation of bioink printability [3], which is the first comprehensive review of bioink printability based on the physical point of view during inkjet printing, laser printing, and microextrusion. In the review, authors characterized the general definition of the bioink printability as the controllable formation of well-defined droplets/jets/filaments and/or the morphology and shape fidelity of deposited building blocks. The core of this review is that authors used a series of self-consistent timescales and dimensionless quantities (Table 1) to physically understand and evaluate bioink printability under different bioprinting techniques. Authors described the unity mechanism of all bioprinting techniques that when a small amount of bioink is ejected out during bioprinting, the forming jet may maintain its shape to form a continuous bioink filament during microextrusion or break up into droplet(s) during inkjet printing or laser printing due to hydrodynamic instability, which only depends on printing conditions and bioink material properties rather than printing devices.

As shown in Table 1, authors utilized three timescales to characterize the jet formation, capillary thinning, and/or breakup of free surface liquid filaments during bioprinting 
Table 1 Dimensionless numbers and ratios and timescales for bioprinting

\begin{tabular}{|c|c|c|c|c|c|}
\hline \multicolumn{3}{|c|}{ Dimensionless numbers for bioprinting } & \multicolumn{3}{|c|}{ Dimensionless ratios for bioprinting } \\
\hline Dimensionless numbers & Definition & Physical interpretation & Dimensionless ratios & Definition & Physical interpretation \\
\hline $\begin{array}{l}\mathrm{Z} \text { or } \mathrm{J} \text { number (inverse of } \\
\text { Oh number) }\end{array}$ & $\frac{\sqrt{\rho \sigma R}}{\eta_{0}}$ & $\begin{array}{l}\text { The ratio of inertial to } \\
\text { viscous effects }\end{array}$ & $\operatorname{Pr}$ & $\frac{L^{2}}{16 A}$ & $\begin{array}{l}\text { The circularity of the cross } \\
\text { section of printed fila- } \\
\text { ments }\end{array}$ \\
\hline Ohnesorge number $(\mathrm{Oh})$ & $\frac{\eta_{0}}{\sqrt{\rho \sigma R}}$ & $\begin{array}{l}\text { The ratio of viscous to } \\
\text { inertial effects }\end{array}$ & Dispensing velocity ratio & $\nu_{\text {path }} / \nu_{\text {out }}$ & $\begin{array}{l}\text { The degree of adaptability } \\
\text { between the dispensing } \\
\text { speed and bioink feed } \\
\text { rate }\end{array}$ \\
\hline $\begin{array}{l}\text { Elasto-capillary number } \\
(\mathrm{Ec})\end{array}$ & $\frac{\lambda \sigma}{\eta_{0} R}$ & $\begin{array}{l}\text { The ratio of elastic to } \\
\text { viscous effects for flows } \\
\text { with non-constant stretch } \\
\text { history }\end{array}$ & Storage modulus ratio & $G_{\text {bath }}^{\prime} / G_{\text {ink }}^{\prime}$ & $\begin{array}{l}\text { The effects of material } \\
\text { properties on filament } \\
\text { formation }\end{array}$ \\
\hline $\begin{array}{l}\text { Intrinsic Deborah number } \\
(\text { De0) }\end{array}$ & $\sqrt{\frac{\lambda^{2} \sigma}{\rho R^{3}}}$ & $\begin{array}{l}\text { The ratio of elastic to iner- } \\
\text { tial effects }\end{array}$ & \multicolumn{3}{|c|}{ Three main timescales for bioprinting of viscoelastic bioinks } \\
\hline Weber number (We) & $\frac{\rho R U^{2}}{\sigma}$ & $\begin{array}{l}\text { The ratio of inertial to } \\
\text { capillary effects }\end{array}$ & Timescales & Definition & Physical interpretation \\
\hline Reynolds number (Re) & $\frac{\rho U R}{\eta_{0}}$ & $\begin{array}{l}\text { The ratio of inertial to } \\
\text { viscous effects }\end{array}$ & Visco-capillary timescale & $t_{v}=\eta_{0} R / \sigma$ & \\
\hline Capillary number $(\mathrm{Ca})$ & $\frac{\eta_{0} U}{\sigma}$ & $\begin{array}{l}\text { The ratio of viscous to } \\
\text { capillary effects }\end{array}$ & $\begin{array}{l}\text { Inertio-capillary or Ray- } \\
\text { leigh timescale }\end{array}$ & $t_{c}=\sqrt{\rho R^{3} / \sigma}$ & \\
\hline Weissenberg number $(\mathrm{Wi})$ & $\frac{\lambda U}{R}$ & $\begin{array}{l}\text { The ratio of elastic to vis- } \\
\text { cous effects for flow with } \\
\text { constant stretch history }\end{array}$ & The longest relaxation time & $\lambda$ & $\begin{array}{l}\text { Characteristic time that } \\
\text { relates to the motion of } \\
\text { polymer chains }\end{array}$ \\
\hline Oldroyd number (Od) & $\frac{\tau_{y} d^{n}}{K U_{\text {nozzle }}^{n}}$ & $\begin{array}{l}\text { Dimensions of the yielded } \\
\text { areas of a Herschel-Bulk- } \\
\text { ley fluid flowing around a } \\
\text { cylinder }\end{array}$ & & & \\
\hline
\end{tabular}

$\eta_{0}$ zero-shear viscosity, $R$ characteristic length scale for the flow, $\sigma$ surface tension, $\rho$ density, $\lambda$ characteristic time, $U$ characteristic velocity of the flow, Unozzle print speed of nozzle, $d$ nozzle outer diameter, $K$ consistency index, $\tau_{\mathrm{y}}$ yield stress, $n$ flow index, $L$ perimeter of hole of printed mesh, $A$ area of hole of printed mesh, $\nu_{\text {out }}$ dispensing velocity, $\nu_{\text {path }}$ : path speed, $G_{\text {bath }}^{\prime}$ storage modulus of support bath materials, $G_{\text {inkl }}^{\prime}$ storage modulus of bioinks

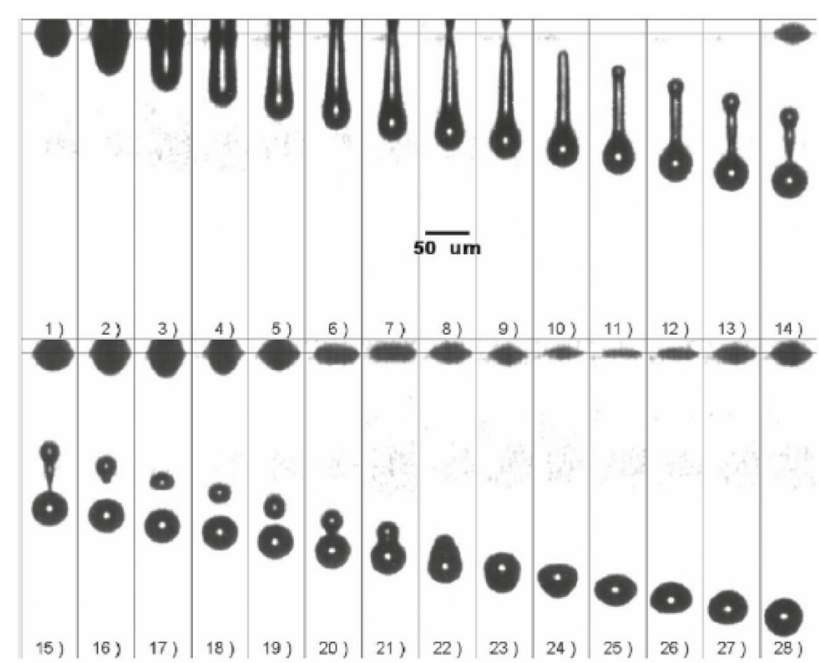

Fig. 1 Representative images of successful droplet formation process during DOD inkjet printing of Newtonian glycerol solutions [4]. Reprinted with permission from Ref. [4], Copyright 2006, American Institute of Physics of viscoelastic bioinks, including visco-capillary timescale $\left(t_{\mathrm{v}}\right)$, inertio-capillary or Rayleigh timescale $\left(t_{\mathrm{c}}\right)$, and the longest relaxation time $(\lambda)$. Moreover, authors classified dimensionless quantities into two types: (1) dimensionless numbers, which are derived from any physical system using dimensional analysis and (2) dimensionless ratios, which are obtained by grouping and canceling out the dimensions of certain system parameters of interest. More importantly, authors revealed the relationship between timescales and dimensionless numbers such as the Ohnesorge number $\mathrm{Oh}=t_{\mathrm{v}} / t_{\mathrm{c}}$, the elasto-capillary number $\mathrm{Ec}=\lambda / t_{\mathrm{v}}$, and the intrinsic Deborah number $\mathrm{De}_{0}=\lambda / t_{\mathrm{c}}$. Also, authors reviewed several dimensionless ratios (Table 1) proposed in previous studies for printability evaluation, e.g., a dimensionless ratio $\operatorname{Pr}=\frac{L^{2}}{16 A}$ has been proposed to quantify the circularity of printed filaments [2].

In this review, authors focused on two major categories of 3D bioprinting: droplet-based and filament-based, respectively. The bioink printability during inkjet bioprinting is discussed with related dimensionless numbers and phase diagrams for Newtonian and non-Newtonian bioinks. For 
Fig. 2 A Four pinch-off types during DOD printing of alginate solutions: a front pinching, $\mathbf{b}$ hybrid pinching, c exit pinching, and $\mathbf{d}$ middle pinching [5]. Reprinted with permission from Ref. [5], Copyright 2017, American Chemical Society

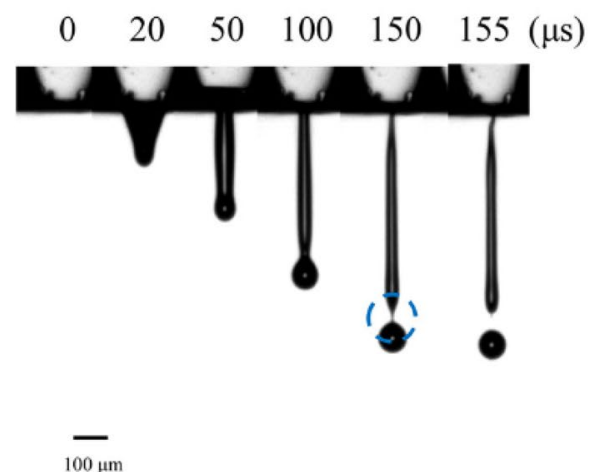

(a)

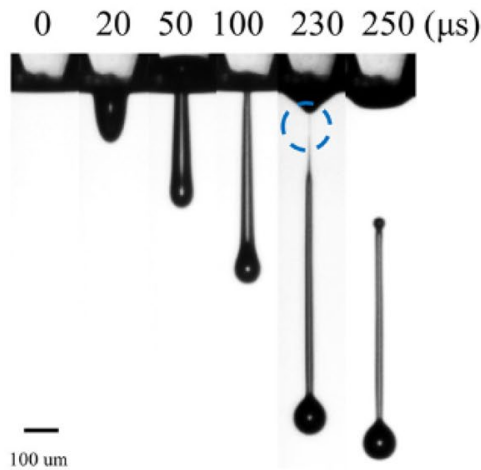

(c)

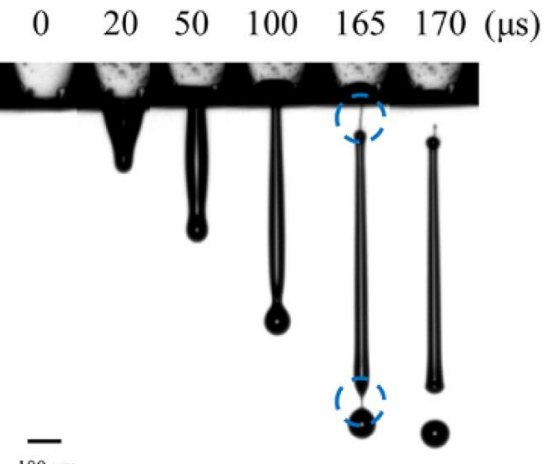

(b)

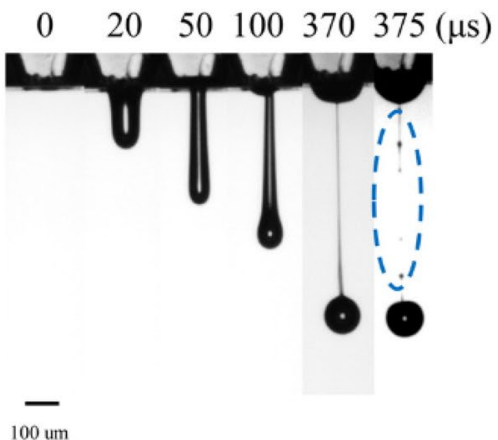

(d)

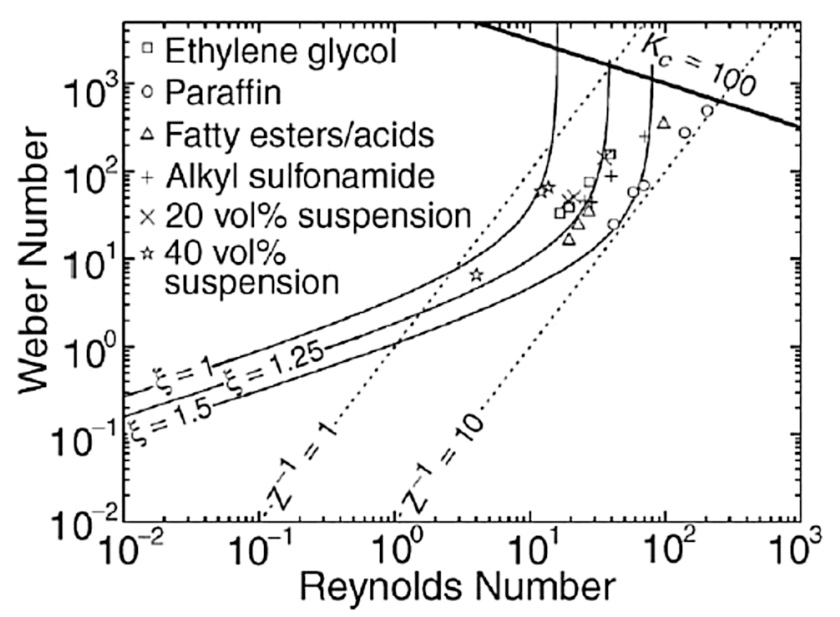

Fig. 3 Example phase diagram during DOD inkjet printing of particle-filled inks [6]. The solid curves indicate contours of equal maximum droplet spreading. The dotted lines indicate the boundaries of fluid properties typical of inkjet printers, and the bold line in the upper-right corner indicates the onset of splashing. Reprinted with permission from Ref. [6], Copyright 2003, Cambridge University Press

Newtonian bioinks, the successful droplet formation usually consists of liquid ejection and stretching, breakup/pinch-off, contraction and breakup of tails, and recombination of primary and satellite droplets (Fig. 1). For non-Newtonian bioinks, four breakup types, namely front pinching, hybrid pinching, exit pinching, and middle pinching, have been identified based on the first pinch-off location (Fig. 2). Moreover, cell-laden bioink has a smaller ejected fluid volume, lower droplet velocity, and longer breakup time compared to a hard bead (polystyrene)-laden suspension.

Authors constructed phase diagrams based on different groups of dimensionless numbers to physically understand the bioink printability during inkjet bioprinting. The $Z$ number (the inverse of Ohnesorge number) and capillary number $(\mathrm{Ca})$ were used along with the Weber number (We) to capture printing conditions' influence by phase diagrams of Newtonian bioinks (Fig. 3). The Weissenberg (Wi) and Deborah (De) numbers were utilized for the evaluation of non-Newtonian bioinks printability in the phase diagram (Fig. 4). Additionally, authors express the essential future work, including revealing the underlying physics of the breakup of fluid jets by investigating the formation process of viscoelastic solutions as well as cell-laden viscoelastic, and constructing unified phase diagrams for bioink printability evaluation based on material properties and operating conditions.

Authors also comprehensively reviewed the studies about bioink printability for extrusion-based bioprinting, including self-supporting in situ rapid solidification and support 


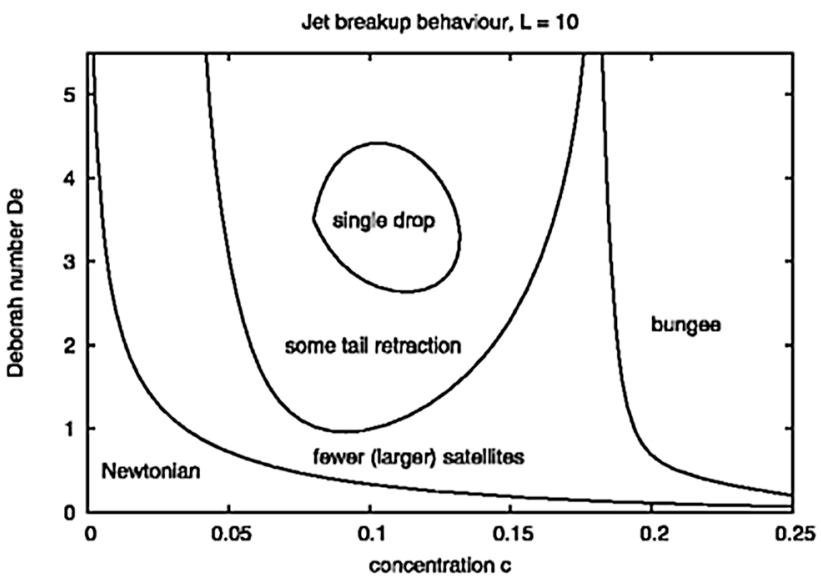

(a)

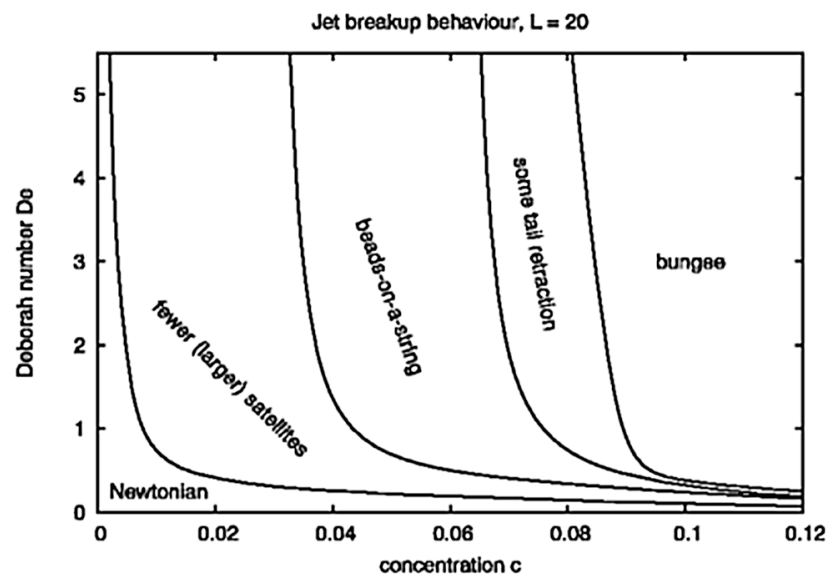

(b)

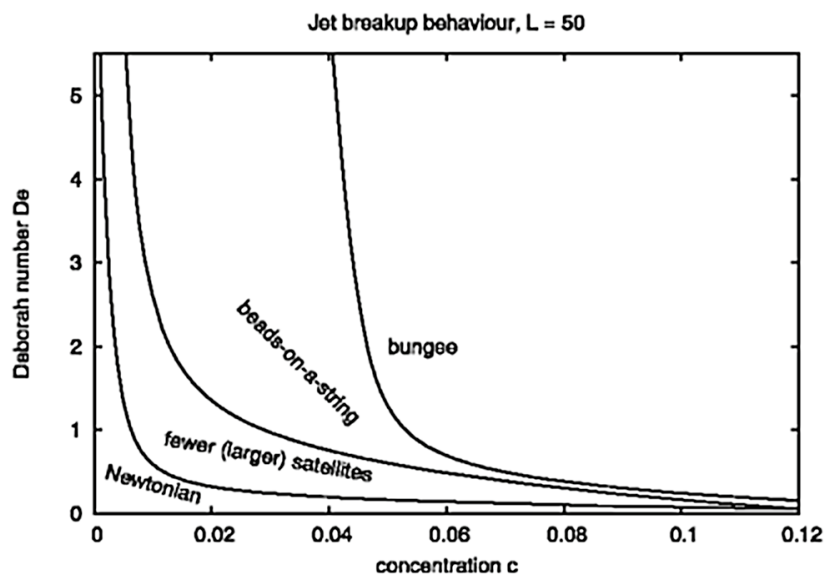

(c)

Fig. 4 Phase diagrams gained via numerical simulations showing the jet breakup behavior in different regions of the viscoelastic parameter space, a $L=10$, b $L=20$, c $L=50$. In which, c and L represents the concentration and molecule extensibility of non-Newtonian inks, respectively [7]. Reprinted with permission from Ref. [7], Copyright 2010, Springer Nature

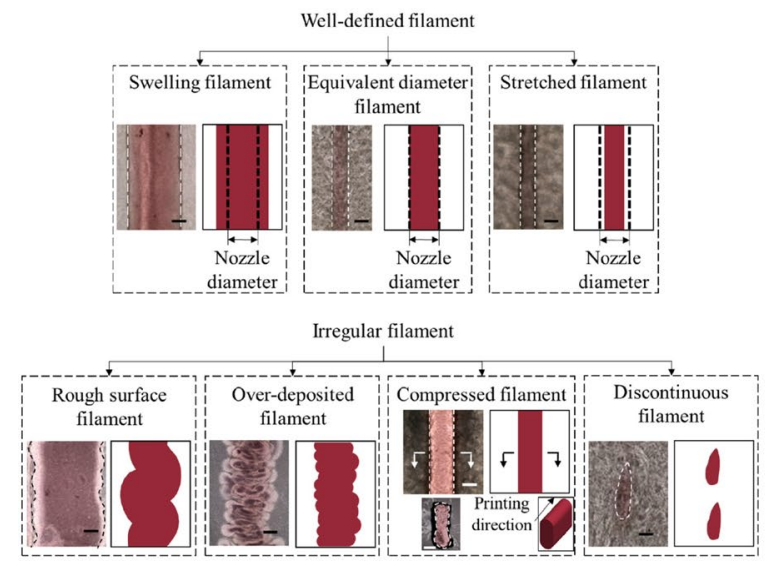

(a)

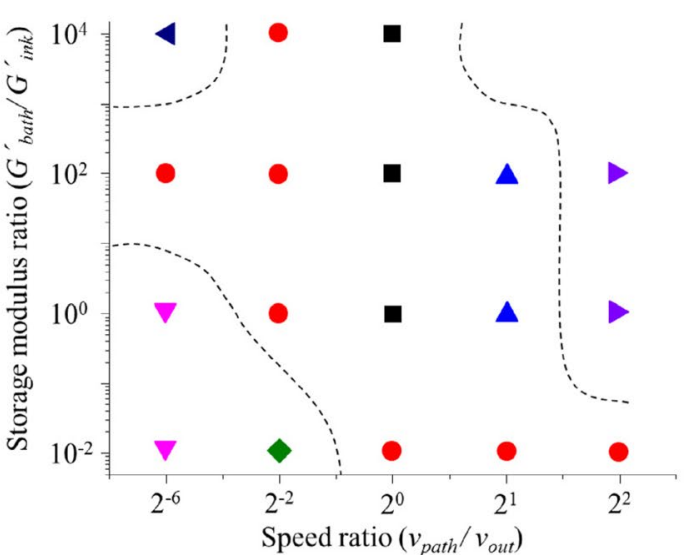

(b)

Fig. 5 a Different types of filaments during support bath-enabled printing. b Phase diagram during support bath-enabled printing [8]. Reprinted with permission from Ref. [8], Copyright 2017, Elsevier 
bath-enabled fabrication. During self-supporting rapid solidification printing, operating conditions usually determine the filament size and printing resolution, including nozzle diameter, dispensing pressure, pathway height, path width, and print speed. In support bath-enabled bioprinting, authors focused more on the studies of the influences of support bath materials on the bioink filament formation. For example, authors reviewed seven possible filament types during extrusion printing of alginate-gelation blends in nanoclay support baths by varying the materials properties and operating conditions (Fig. 5a) and constructed a phase diagram (Fig. 5b) for the printability using dimensionless ratios [speed ratio $\left(v=v_{\text {path }} / v_{\text {out }}\right)$ and storage modulus ratio $\left.\left(G_{\text {bath }}^{\prime} / G_{\text {ink }}^{\prime}\right)\right]$. Also, they reviewed Oldroyd number $(\mathrm{Od})$ to characterize the dimensions of the yield areas of a Herschel-Bulkley fluid flowing around a cylinder.

This review for the first time presents a comprehensive summary of the recent advances of bioink printability studies based on physical understanding. The phase diagrams have been constructed based on different groups of dimensionless numbers to effectively summarize the knowledge of various bioink printability studies. It is also noticed that since digital light processing (DLP)-based bioprinting is considered as the indirect bioprinting, the printability of DLP-based bioprinting has not been reviewed yet, which would be a promising and necessary topic for authors.

\section{Compliance with ethical standards}

Conflict of interest J.Y, D.Z and J.L declare that they have no conflict of interest.
Ethical approval This review does not contain any studies with human or animal subjects performed by any of the authors.

Open Access This article is distributed under the terms of the Creative Commons Attribution 4.0 International License (http://creativeco mmons.org/licenses/by/4.0/), which permits unrestricted use, distribution, and reproduction in any medium, provided you give appropriate credit to the original author(s) and the source, provide a link to the Creative Commons license, and indicate if changes were made.

\section{References}

1. Placone JK, Engler AJ (2018) Recent advances in extrusion-based 3D printing for biomedical applications. Adv Healthc Mater 7:1701161

2. Ouyang L, Yao R, Zhao Y, Sun W (2016) Effect of bioink properties on printability and cell viability for 3D bioplotting of embryonic stem cells. Biofabrication 8:035020

3. Zhang Z, Jin Y, Yin J, Xu C, Xiong R, Christensen K, Ringeisen BR, Chrisey DB, Huang Y (2018) Evaluation of bioink printability for bioprinting applications. Appl Phys Rev 5:041304

4. Dong H, Carr WW, Morris JF (2006) An experimental study of drop-on-demand drop formation. Phys Fluids 18:072102

5. Xu C, Zhang Z, Fu J, Huang Y (2017) Study of pinch-off locations during drop-on-demand inkjet printing of viscoelastic alginate solutions. Langmuir 33:5037-5045

6. Derby B, Reis N (2003) Inkjet printing of highly loaded particulate suspensions. MRS Bull 28:815-818

7. Morrison NF, Harlen OG (2010) Viscoelasticity in inkjet printing. Rheol Acta 49:619-632

8. Jin Y, Chai W, Huang Y (2017) Printability study of hydrogel solution extrusion in nanoclay yield-stress bath during printingthen-gelation biofabrication. Mater Sci Eng C 80:313 